\title{
Prof. Dr. med. Lutz Jani zum 80. Geburtstag
}

\begin{abstract}
„Der neue Mann kommt aus Basel“, das war 1982 die Überschrift des Mannheimer Morgen, als Prof. Dr. med. Lutz Jani die Leitung der Orthopädischen Universitätsklinik in Mannheim übernahm. Es ist uns deshalb eine innere Verpflichtung und ganz besondere Freude, Prof. Dr. Lutz Jani mit einem Symposium* zu seinem 80. Geburtstag zu gratulieren. Der Blick zurück und der Blick nach vorne sollen ihn als Mensch feiern und seine Verdienste um die Patienten und das Fach Orthopädie würdigen.
\end{abstract}

Nach den Wirren des zweiten Weltkrieges, die er in Dresden bis 1945 erlebte hatte, studierte Jani von 1954 bis 1960 an den Universitäten in München und Freiburg Humanmedizin. Nach seiner Medizinalassistentenzeit in der Inneren Medizin, der Pathologie und der Gynäkologie erhielt er 1962 seine Approbation. Nach seiner Weiterbildung zum Orthopäden bei den Professoren Lange und Witt an der Orthopädischen Universitätsklinik in München erwarb er 1966 seine Facharztanerkennung und wurde zum stellvertretenden Oberarzt ernannt. In diese Zeit fallen auch die ersten wissenschaftlichen Publikationen von Prof. Jani. Der thematische Schwerpunkt dieser Arbeiten lag bei der operativen Therapie der Hüftdysplasie und typisch für Prof. Jani, bereits damals, bei den langfristigen Ergebnissen der operierten Kinder.

Im Jahr 1969 wechselte Prof. Jani an die Orthopädische Universitätsklinik Basel und wurde zum Oberarzt dieser renommierten Klinik ernannt. Es folgten 1973 die Habilitation und die Ernennung zum stellvertretenden Leiter der Klinik im Jahr 1975. Die außerplanmäßige Professur wurde ihm 1980 verliehen. Schwerpunkt dieser 11-jährigen Tätigkeit in Basel war die Kinderorthopädie mit insgesamt 96 wissenschaftlichen Publikationen und ungezählten Vorträgen. Die Ergebnisse seiner Forschung haben sich nachhaltig und direkt auf die klinische Versorgung von Kindern mit einer Hüftdysplasie oder einem Morbus Perthes ausgewirkt. Mit seinen langfristigen Untersuchungsergebnissen konnte Prof. Jani zeigen, dass varisierende, derotierende, intertrochantäre Osteotomien, auf Grund der Neigung zur spontanen Korrektur in der präpubertären Wachstumsphase, oft vermeidbar sind.

Den Ruf nach Mannheim erhielt Prof. Dr. med. Lutz Jani 1982, mit dem Ordinariat verbunden ist die Leitung der Orthopädischen Universitätsklinik, die damals noch auf den Lindenhof ausgelagert war. Unter Leitung von Prof. Jani wurde „der Lindenhof" zu einem Markenzeichen in Mannheim und seiner weiteren Umgebung. Mit der Weiterentwicklung des Faches Orthopädie und dem ständig wachsenden, operativen Spektrum der Klinik wuchsen rasch auch die Anforderungen an Räumlichkeiten und Operationssäle. Deshalb erfolgte 1995 der Umzug der Klinik in den Neubau der Orthopädie am Universitätsklinikum am Neckar. Durch den Umzug verfügte die Klinik jetzt auch über einen eigenständigen Forschungsbereich mit dem Schwerpunkt der Biomechanik. Damit konnte Prof. Jani sich neben seinem Steckenpferd der Kinderorthopädie nun auch klinisch, wissen- schaftlich und experimentell um die Endoprothetik, seiner zweiten Vorliebe, kümmern. Die Ergebnisse seiner wissenschaftlichen Arbeit sind in 38 Originalarbeiten heute noch verfügbar, relevant und auch lesenswert.

Neben seiner klinischen und wissenschaftlichen Arbeit engagierte sich Prof. Jani auch in der ärztlichen Selbstverwaltung und den Wissenschaftlichen Fachgesellschaften. Als Präsident der DGOOC richtete er 1992 den Kongress der Wissenschaftlichen Fachgesellschaft in Mannheim aus. Es folgten die Kongresse der Rheumaorthopäden (1992) und der Kinderorthopäden (1994). Nach seinem Präsidentenamt blieb Prof. Jani im Vorstand der DGOOC und wurde von 1999 bis 2002 ihr Generalsekretär. In dieser Funktion kümmerte er sich intensiv um die Zusammenführung von Orthopädie und Unfallchirurgie, die ärztliche Weiterbildung und die externe Qualitätssicherung. Sein ungeteilter Einsatz für das Fach Orthopädie fand Anerkennung in den Ehrenmitgliedschaften der Deutschen und der Tschechischen Gesellschaften für Orthopädie und Unfallchirurgie, sowie der Verleihung des Lexer-Preises im Jahr 2000.

Diese, rein auf den Beruf bezogene Laudatio, greift aber sicherlich zu kurz, denn sie vernachlässigt die Persönlichkeit von Prof. Jani. Stets ausgeglichen, freundlich und zugewandt für Patienten und Mitarbeiter, in der fachlichen Diskussion intellektuell präzise, aber stets ein offenes Ohr und eine ehrliche Antwort, die durch ein freundliches Lächeln begleitet und auch beim Widerspruch geschätzt wird, das zeichnet Prof. Jani bis heute aus.

Wir gratulieren Prof. Dr. med. Lutz Jani zusammen mit allen Kollegen, Mitarbeitern und Freunden und wünschen alles Gute, Glück und Gesundheit für seinen weiteren Lebensweg.

Prof. Dr. med. Hanns-Peter Scharf Universitätsmedizin Mannheim Orthopädisch-Unfallchirurgisches Zentrum

Theodor-Kutzer-Ufer 1-3

68167 Mannheim

\footnotetext{
* Das Symposium zum 80. Geburtstag von Prof. Dr. med. Lutz Jani „Einst und Jetzt, Wandel in Orthopädie \& Unfallchirurgie“ fand am Freitag, 30.01.2015 in der Universitätsmedizin Mannheim statt.
} 